\title{
Nonhomogeneous Boundary Value Problem for One-Dimensional Compressible Viscous Micropolar Fluid Model: Regularity of the Solution
}

\author{
Nermina Mujaković \\ Department of Mathematics, Faculty of Philosophy, University of Rijeka, 51000 Rijeka, Croatia
}

Correspondence should be addressed to Nermina Mujaković, mujakovic@inet.hr

Received 22 June 2008; Accepted 22 October 2008

Recommended by Michel Chipot

An initial-boundary value problem for 1D flow of a compressible viscous heat-conducting micropolar fluid is considered; the fluid is thermodynamically perfect and polytropic. Assuming that the initial data are Hölder continuous on ]0,1[ and transforming the original problem into homogeneous one, we prove that the state function is Hölder continuous on $] 0,1[\times] 0, T[$, for each $T>0$. The proof is based on a global-in-time existence theorem obtained in the previous research paper and on a theory of parabolic equations.

Copyright ( 2008 Nermina Mujaković. This is an open access article distributed under the Creative Commons Attribution License, which permits unrestricted use, distribution, and reproduction in any medium, provided the original work is properly cited.

\section{Introduction}

In this paper, we consider a nonstationary 1D flow of a compressible viscous and heatconducting micropolar fluid, being in a thermodynamical sense perfect and polytropic. In [1-3], we considered the problem with homogeneous boundary conditions.

Here we study, as in $[4,5]$, the case of nonhomogeneous boundary conditions for velocity and microrotation which is called in gas dynamics "problem on piston" (see [6]). Assuming that the initial data are Hölder continuous on ]0,1[ and transforming the original problem into homogeneous one, we prove that, for each $T>0$, the mass density, velocity, microrotation velocity, and temperature are Hölder continuous on $] 0,1[\times] 0, T[$. The proof is based on a global-in-time existence theorem [5] and on a theory of parabolic equations [7]. We use some ideas of Antontsev et al. [8] applied to the case of classical fluid with homogeneous boundary conditions, results from [3] as well and some inequalities for Hölder norms obtained by the Nirenberg-Gagliardo inequality.

\section{Statement of the problem and its equivalent setting}

Let $\rho, v, \omega$, and $\theta$ denote, respectively, the mass density, velocity, microrotation velocity, and temperature of the fluid in the Lagrangean description. Then the problem which we consider 
has the formulation as follows [1]:

$$
\begin{gathered}
\frac{\partial \rho}{\partial t}+\rho^{2} \frac{\partial v}{\partial x}=0 \\
\frac{\partial v}{\partial t}=\frac{\partial}{\partial x}\left(\rho \frac{\partial v}{\partial x}\right)-K \frac{\partial}{\partial x}(\rho \theta), \\
\rho \frac{\partial \omega}{\partial t}=A\left[\rho \frac{\partial}{\partial x}\left(\rho \frac{\partial \omega}{\partial x}\right)-\omega\right], \\
\rho \frac{\partial \theta}{\partial t}=-K \rho^{2} \theta \frac{\partial v}{\partial x}+\rho^{2}\left(\frac{\partial v}{\partial x}\right)^{2}+\rho^{2}\left(\frac{\partial \omega}{\partial x}\right)^{2}+\omega^{2}+D \rho \frac{\partial}{\partial x}\left(\rho \frac{\partial \theta}{\partial x}\right)
\end{gathered}
$$

in $] 0,1[\times] 0, T[, T>0$, where $K, A$, and $D$ are positive constants. Equations (2.1)(2.4) are, respectively, local forms of the conservations laws for the mass, momentum, momentum moment, and energy. We take the following nonhomogeneous initial and boundary conditions:

$$
\begin{gathered}
\rho(x, 0)=\rho_{0}(x), \\
v(x, 0)=v_{0}(x) \\
\omega(x, 0)=\omega_{0}(x) \\
\theta(x, 0)=\theta_{0}(x) \\
v(0, t)=\mu_{0}(t), \quad v(1, t)=\mu_{1}(t), \\
\omega(0, t)=v_{0}(t), \quad \omega(1, t)=v_{1}(t), \\
\frac{\partial \theta}{\partial x}(0, t)=\frac{\partial \theta}{\partial x}(1, t)=0
\end{gathered}
$$

for $x \in \Omega=] 0,1[, t \in] 0, T\left[\right.$. Here $\rho_{0}, v_{0}, \omega_{0}, \theta_{0}, \mu_{0}, \mu_{1}, v_{0}$, and $v_{1}$ are given functions. We assume the compatibility conditions

$$
\begin{gathered}
v_{0}(0)=\mu_{0}(0), \quad v_{0}(1)=\mu_{1}(0), \\
\omega_{0}(0)=v_{0}(0), \quad \omega_{0}(1)=v_{1}(0), \\
\frac{\partial \theta_{0}}{\partial x}(0)=\frac{\partial \theta_{0}}{\partial x}(1)=0,
\end{gathered}
$$

and the inequalities

$$
0<m \leq \rho_{0}(x) \leq M, \quad m \leq \theta_{0}(x) \leq M \quad \text { for } x \in \Omega,
$$

where $m, M \in R^{+}$. We assume also that there exists a constant $\delta>0$ such that

$$
\left.l(t)=\int_{0}^{1} \frac{1}{\rho_{0}(x)} d x+\int_{0}^{t}\left[\mu_{1}(\tau)-\mu_{0}(\tau)\right] d \tau \geq \delta, \quad t \in\right] 0, T[.
$$


In the previous work [5] we proved that for

$$
\begin{aligned}
& \mu_{0}, \mu_{1}, v_{0}, v_{1} \in H^{2}(] 0, T[), \\
& \rho_{0}, v_{0}, \omega_{0}, \theta_{0} \in H^{1}(\Omega),
\end{aligned}
$$

the problems (2.1)-(2.4) have a unique generalized solution

$$
\begin{gathered}
\left.(x, t) \longrightarrow(\rho, v, \omega, \theta)(x, t), \quad(x, t) \in Q_{T}=\Omega \times\right] 0, T[, \\
\rho \in L^{\infty}\left(0, T ; H^{1}(\Omega)\right) \cap H^{1}\left(Q_{T}\right), \quad \inf _{Q_{T}} \rho>0, \\
v, \omega, \theta \in L^{\infty}\left(0, T ; H^{1}(\Omega)\right) \cap H^{1}\left(Q_{T}\right) \cap L^{2}\left(0, T ; H^{2}(\Omega)\right),
\end{gathered}
$$

that satisfies (2.1)-(2.4) a.e. in $Q_{T}$ and conditions (2.5)-(2.11) in the sense of traces. Moreover,

$$
\theta>0 \text { in } \bar{Q}_{T} .
$$

From embedding and interpolation theorems (e.g., [9]) one can conclude that from (2.19) and (2.20) it follows:

$$
\begin{gathered}
\rho \in L^{\infty}(0, T ; C(\bar{\Omega})) \cap C\left([0, T], L^{2}(\Omega)\right), \\
v, \omega, \theta \in L^{2}\left(0, T ; C^{1}(\bar{\Omega})\right) \cap C\left([0, T], H^{1}(\Omega)\right), \\
v, \omega, \theta \in C\left(\bar{Q}_{T}\right) .
\end{gathered}
$$

Now, instead of the velocity $v$ and microrotation $\omega$ we introduce new functions $V$ and $W$ in order to obtain a problem with the homogeneous boundary conditions.

Notice that using (2.9) from (2.1) we get

$$
\left.\int_{0}^{1} \frac{d x}{\rho(x, t)}=l(t), \quad t \in\right] 0, T[,
$$

where the function $l$ is defined by (2.16). We introduce the functions

$$
\begin{aligned}
& v_{1}(x, t)=\frac{\mu(t)}{l(t)} \int_{0}^{x} \frac{d \xi}{\rho(\xi, t)}+\mu_{0}(t) \\
& \omega_{1}(x, t)=\frac{v(t)}{l(t)} \int_{0}^{x} \frac{d \xi}{\rho(\xi, t)}+v_{0}(t) \text { on } Q_{T}
\end{aligned}
$$

where $\mu(t)=\mu_{1}(t)-\mu_{0}(t)$ and $v(t)=v_{1}(t)-v_{0}(t)$. It is evident that

$$
\begin{gathered}
v_{1}(0, t)=\mu_{0}(t), \quad v_{1}(1, t)=\mu_{1}(t), \\
\left.\omega_{1}(0, t)=v_{0}(t), \quad \omega_{1}(1, t)=v_{1}(t), \quad t \in\right] 0, T[
\end{gathered}
$$


Inserting

$$
V(x, t)=v(x, t)-v_{1}(x, t), \quad W(x, t)=\omega(x, t)-\omega_{1}(x, t)
$$

into (2.1)-(2.4) we get the following equivalent system:

$$
\begin{gathered}
\frac{\partial \rho}{\partial t}+\rho^{2} \frac{\partial V}{\partial x}+\frac{\mu}{l} \rho=0 \\
\frac{\partial V}{\partial t}=\frac{\partial}{\partial x}\left(\rho \frac{\partial V}{\partial x}\right)-K \frac{\partial}{\partial x}(\rho \theta)-\frac{\partial v_{1}}{\partial t} \\
\rho \frac{\partial W}{\partial t}=A\left[\rho \frac{\partial}{\partial x}\left(\rho \frac{\partial W}{\partial x}\right)-\omega_{1}-W\right]-\rho \frac{\partial \omega_{1}}{\partial t} \\
\rho \frac{\partial \theta}{\partial t}=-K \rho^{2} \theta \frac{\partial V}{\partial x}-K \rho \theta \frac{\mu}{l}+\rho^{2}\left(\frac{\partial V}{\partial x}\right)^{2}+2 \rho \frac{\partial V}{\partial x} \frac{\mu}{l}+\left(\frac{\mu}{l}\right)^{2} \\
+\rho^{2}\left(\frac{\partial W}{\partial x}\right)^{2}+2 \rho \frac{\partial W}{\partial x} \frac{v}{l}+\left(\frac{v}{l}\right)^{2}+\left(W+\omega_{1}\right)^{2}+D \rho \frac{\partial}{\partial x}\left(\rho \frac{\partial \theta}{\partial x}\right),
\end{gathered}
$$

with the homogeneous boundary conditions

$$
\begin{gathered}
V(0, t)=V(1, t)=0, \quad W(0, t)=W(1, t)=0, \\
\frac{\partial \theta}{\partial x}(0, t)=\frac{\partial \theta}{\partial x}(1, t)=0
\end{gathered}
$$

for $t \in] 0, T[$ and initial conditions

$$
\begin{gathered}
\rho(x, 0)=\rho_{0}(x), \quad V(x, 0)=V_{0}(x), \\
W(x, 0)=W_{0}(x), \quad \theta(x, 0)=\theta_{0}(x),
\end{gathered}
$$

for $x \in \Omega$, where

$$
\begin{aligned}
& V_{0}(x)=v_{0}(x)-\frac{\mu(0)}{l(0)} \int_{0}^{x} \frac{1}{\rho_{0}(\xi)} d \xi-\mu_{0}(0), \\
& W_{0}(x)=\omega_{0}(x)-\frac{v(0)}{l(0)} \int_{0}^{x} \frac{1}{\rho_{0}(\xi)} d \xi-v_{0}(0)
\end{aligned}
$$

are known functions. In the article [5], we proved that the problems (2.30)-(2.37) have a unique generalized solution $(\rho, V, W, \theta)$ in the domain $Q_{T}$ with property (2.21) as well. Moreover, we obtained that

$$
v_{1}, \omega_{1} \in L^{\infty}\left(0, T ; H^{2}(\Omega)\right), \quad \frac{\partial v_{1}}{\partial t}, \frac{\partial \omega_{1}}{\partial t} \in L^{\infty}\left(0, T ; L^{2}(\Omega)\right)
$$


In the following $C^{k+\alpha}\left(\bar{Q}_{T}\right)(k \in N \cup\{0\}, 0<\alpha<1)$ is the Banach space of functions of the class $C^{k}\left(\bar{Q}_{T}\right)$, having $k$ th derivatives Hölder continuous with the exponent $\alpha$ on $\bar{Q}_{T}$; the norm is defined by

$$
|f|_{k+\alpha, Q_{T}}=\sum_{m+j=0}^{k}\left|D_{x}^{m} D_{t}^{j} f\right|_{0, Q_{T}}+\sum_{m+j=k} H^{\alpha}\left(D_{x}^{m} D_{t}^{j} f\right),
$$

where

$$
H^{\alpha}(f)=\sup _{y, z \in Q_{T}} \frac{|f(y)-f(z)|}{|y-z|^{\alpha}}
$$

and $|\cdot|_{0, Q_{T}}$ is the norm on $C\left(\bar{Q}_{T}\right) ; D_{x}^{m}$ and $D_{t}^{j}$ are, respectively, the $m$ th derivatives with respect to $x$ and the $j$ th derivatives with respect to $t . C^{k+\alpha, m+\beta}\left(\bar{Q}_{T}\right)(k, m \in N \cup\{0\}, 0<\alpha, \beta<1)$ is the Banach space of functions which have $k$ th derivatives with respect to $x$ and $m$ th derivatives with respect to $t$ Hölder continuous on $\bar{Q}_{T}$. The norm is defined by

$$
|f|_{k+\alpha, m+\beta, Q_{T}}=\sum_{l=0}^{k}\left|D_{x}^{l} f\right|_{0, Q_{T}}+\sum_{j=1}^{m}\left|D_{t}^{j} f\right|_{0, Q_{T}}+H_{x}^{\alpha}\left(D_{x}^{k} f\right)+H_{t}^{\beta}\left(D_{x}^{k} f\right)+H_{x}^{\alpha}\left(D_{t}^{m} f\right)+H_{t}^{\beta}\left(D_{t}^{m} f\right),
$$

where

$$
\begin{aligned}
& H_{x}^{\alpha}(f)=\sup _{\left(x_{1}, t\right),\left(x_{2}, t\right) \in Q_{T}} \frac{\left|f\left(x_{1}, t\right)-f\left(x_{2}, t\right)\right|}{\left|x_{1}-x_{2}\right|^{\alpha}}, \\
& H_{t}^{\beta}(f)=\sup _{\left(x, t_{1}\right),\left(x, t_{2}\right) \in Q_{T}} \frac{\left|f\left(x, t_{1}\right)-f\left(x, t_{2}\right)\right|}{\left|t_{1}-t_{2}\right|^{\beta}} .
\end{aligned}
$$

By $C \in \mathbf{R}^{+}$we denote a generic constant, having possibly different values at different places. Also we use some inequalities for Hölder norms obtained by the following NirenbergGagliardo interpolation inequality

$$
|f|_{1 / \mu} \leq|f|_{1 / \lambda}^{(v-\mu) /(v-\lambda)}|f|_{1 / v}^{(\mu-\lambda) /(v-\lambda)},
$$

where $\mu, v, \lambda \in \mathbf{R}$ and $\lambda \leq \mu \leq \nu$. Here, for bounded domain $D \subset \mathbf{R}^{n}$ and $f: D \rightarrow \mathbf{R}$ the norm $|f|_{q}$ is defined by

$$
|f|_{q}= \begin{cases}\|f\|_{L^{q}(D)}, & q>0 \\ |f|_{k+\beta, D}, & q<0\end{cases}
$$

where $k=[-n / q]$ and $\beta=-n / q-k$ (e.g., [8, page 27]). Some of our considerations are very similar or identical to that of [8] or [3]. In these cases we omit proofs or details of proofs, 
making reference to correspondent pages of the book [8] or article [3]; we use the notation $\|\cdot\|=\|\cdot\|_{L^{2}}$.

\section{The main results}

The aim of this paper is to prove the following regularity result.

Theorem 3.1. Let the functions

$$
\begin{gathered}
\mu_{0}, \mu_{1}, v_{0}, v_{1} \in C^{2}([0, T]), \\
\rho_{0} \in C^{1+\alpha}(\bar{\Omega}), \quad v_{0}, \omega_{0}, \theta_{0} \in C^{2+\alpha}(\bar{\Omega}), \quad 0<\alpha<1
\end{gathered}
$$

satisfy the compatibility conditions

$$
\begin{gathered}
\frac{d}{d x}\left(\rho_{0} \frac{d v_{0}}{d x}\right)-K \frac{d}{d x}\left(\rho_{0} \theta_{0}\right)= \begin{cases}\frac{d \mu_{0}(0)}{d t}, & \text { for } x=0 \\
\frac{d \mu_{1}(0)}{d t}, & \text { for } x=1,\end{cases} \\
A\left[\frac{d}{d x}\left(\rho_{0} \frac{d \omega_{0}}{d x}\right)-\frac{\omega_{0}}{\rho_{0}}\right]= \begin{cases}\frac{d v_{0}(0)}{d t}, & \text { for } x=0 \\
\frac{d v_{1}(0)}{d t}, & \text { for } x=1,\end{cases}
\end{gathered}
$$

and (2.12)-(2.16). Then the generalized solution of the problems (2.1)-(2.11) has the properties

$$
\rho \in C^{1+\alpha}\left(\bar{Q}_{T}\right), \quad v, \omega, \theta \in C^{2+\alpha, 1+\alpha / 2}\left(\bar{Q}_{T}\right), \quad 0<\alpha<1 .
$$

Notice that because of (3.1) and (3.2) we have

$$
V_{0}, W_{0} \in C^{2+\alpha}(\bar{\Omega}), \quad l \in C^{3}([0, T])
$$

and for $t=0$ we can easily conclude that

$$
\left.\left.\left.v_{1}\right|_{t=0^{\prime}} \omega_{1}\right|_{t=0^{\prime}} \frac{\partial v_{1}}{\partial t}\right|_{t=0},\left.\frac{\partial \omega_{1}}{\partial t}\right|_{t=0} \in C^{2+\alpha}(\bar{\Omega})
$$

Now, (2.12), (2.13), (3.3), and (3.4) become the following compatibility conditions for the problems (2.30)-(2.37)

$$
V_{0}(0)=V_{0}(1)=0, \quad W_{0}(0)=W_{0}(1)=0,
$$


Nermina Mujaković

$$
\begin{gathered}
\frac{d}{d x}\left(\rho_{0} \frac{d V_{0}}{d x}\right)-K \frac{d}{d x}\left(\rho_{0} \theta_{0}\right)= \begin{cases}\frac{d \mu_{0}(0)}{d t}, & \text { for } x=0 \\
\frac{d \mu_{1}(0)}{d t}, & \text { for } x=1,\end{cases} \\
\frac{d}{d x}\left(\rho_{0} \frac{d W_{0}}{d x}\right)-\frac{W_{0}}{\rho_{0}}= \begin{cases}\frac{v_{0}(0)}{\rho_{0}}+A^{-1} \frac{d v_{0}(0)}{d t}, & \text { for } x=0 \\
\frac{v_{1}(0)}{\rho_{0}}+A^{-1} \frac{d v_{1}(0)}{d t}, & \text { for } x=1 .\end{cases}
\end{gathered}
$$

In this paper, we will prove the following result first.

Theorem 3.2. Under the assumptions of Theorem 3.1 the problems (2.30)-(2.37) have a generalized solution $(\rho, V, W, \theta)$ in $Q_{T}$ with the properties

$$
\rho \in C^{1+\alpha}\left(\bar{Q}_{T}\right), \quad V, W, \theta \in C^{2+\alpha, 1+\alpha / 2}\left(\bar{Q}_{T}\right), \quad 0<\alpha<1 .
$$

Moreover,

$$
v_{1}, \omega_{1} \in C^{2+\alpha, 1+\alpha / 2}\left(\bar{Q}_{T}\right) .
$$

Theorem 3.1 is an immediate consequence of this result. In the proof of Theorem 3.2 we apply, as in [3], the method of the book [8], where Theorem 3.1 was proved for the classical fluid $(\omega=0)$ with homogeneous boundary conditions.

In that what follows, we assume that the conditions (2.12)-(2.16) and (3.1)-(3.4) are fulfilled.

\section{Some properties of the solution $(\rho, V, W, \theta)$ and functions $v_{1}$ and $\omega_{1}$}

Lemma 4.1. It holds

$$
\frac{\partial^{2} v_{1}}{\partial t^{2}}, \frac{\partial^{2} \omega_{1}}{\partial t^{2}} \in L^{2}\left(0, T ; L^{2}(\Omega)\right)
$$

Proof. Using (2.30) from (2.26) and (2.27) we get

$$
\begin{aligned}
& \frac{\partial v_{1}}{\partial t}=\left[\left(\frac{\mu}{l}\right)^{\prime}+\left(\frac{\mu}{l}\right)^{2}\right] \int_{0}^{x} \frac{1}{\rho} d \xi+\frac{\mu}{l} V+\mu_{0}^{\prime} \\
& \frac{\partial \omega_{1}}{\partial t}=\left[\left(\frac{v}{l}\right)^{\prime}+\frac{\mu \nu}{l^{2}}\right] \int_{0}^{x} \frac{1}{\rho} d \xi+\frac{v}{l} V+v_{0}^{\prime}
\end{aligned}
$$

After differentiating (4.2) with respect to $t$, squaring, integrating over $\Omega$ and taking into account (2.25), (2.30), (3.1), and (3.6) we get

$$
\left\|\frac{\partial^{2} v_{1}}{\partial t^{2}}(t)\right\|^{2} \leq C\left(1+\|V(t)\|^{2}+\left\|\frac{\partial V}{\partial t}(t)\right\|^{2}\right) .
$$


With the help of (2.20) for $V$ we conclude that

$$
\int_{0}^{T}\left\|\frac{\partial^{2} v_{1}}{\partial t^{2}}(\tau)\right\|^{2} d \tau \leq C\left(1+\int_{0}^{T}\|V(\tau)\|^{2} d \tau+\int_{0}^{T}\left\|\frac{\partial V}{\partial t}(\tau)\right\|^{2} d \tau\right) \leq C .
$$

From (4.3) follows the same estimation for $\partial^{2} \omega_{1} / \partial t^{2}$.

Lemma 4.2. The inclusions

$$
\frac{\partial \rho}{\partial t}, \frac{\partial V}{\partial t}, \frac{\partial W}{\partial t}, \frac{\partial \theta}{\partial t} \in L^{\infty}\left(0, T ; L^{2}(\Omega)\right) \cap L^{2}\left(0, T ; H^{1}(\Omega)\right)
$$

hold true.

Proof. Using (2.22) for $\rho$ and (2.20) for $V$ from (2.30) we get immediately that $\partial \rho / \partial t \in$ $L^{\infty}\left(0, T ; L^{2}(\Omega)\right)$. Differentiating $(2.30)$ with respect to $x$, using the inequalities

$$
\begin{gathered}
|f|^{2} \leq C\|f\|\left\|f^{\prime}\right\| \leq C\left\|f^{\prime}\right\|^{2}, \\
\left|f^{\prime}\right|^{2} \leq C\left\|f^{\prime}\right\|\left\|f^{\prime \prime}\right\| \leq C\left\|f^{\prime \prime}\right\|^{2}
\end{gathered}
$$

(valid for a function $f$ or its derivative vanishing at $x=0$ and $x=1),(2.22)$ and (3.1) we obtain

$$
\begin{aligned}
\left\|\frac{\partial^{2} \rho}{\partial x \partial t}(t)\right\|^{2} & \leq C\left(\int_{0}^{1}\left|\frac{\partial \rho}{\partial x}(t)\right|^{2}\left|\frac{\partial V}{\partial x}\right|^{2} d x+\int_{0}^{1}\left|\frac{\partial^{2} V}{\partial x^{2}}\right|^{2} d x+\left|\frac{\mu}{l}\right|^{2} \int_{0}^{1}\left|\frac{\partial \rho}{\partial x}\right|^{2} d x\right) \\
& \leq C\left(\left\|\frac{\partial \rho}{\partial x}(t)\right\|^{2}\left\|\frac{\partial^{2} V}{\partial x^{2}}(t)\right\|^{2}+\left\|\frac{\partial^{2} V}{\partial x^{2}}(t)\right\|^{2}+\left\|\frac{\partial \rho}{\partial x}(t)\right\|^{2}\right) .
\end{aligned}
$$

Taking into account (2.20) and (2.19) we get

$$
\int_{0}^{T}\left\|\frac{\partial^{2} \rho}{\partial x \partial t}(\tau)\right\|^{2} d \tau \leq C .
$$

After differentiating (2.31) with respect to the time variable, multiplying by $\partial V / \partial t$ and integrating by parts over $\Omega$ we obtain

$$
\begin{aligned}
& \frac{1}{2} \frac{d}{d t}\left\|\frac{\partial V}{\partial t}(t)\right\|^{2}+\int_{0}^{1} \rho\left(\frac{\partial^{2} V}{\partial x \partial t}\right)^{2} d x \\
& \quad=-\int_{0}^{1} \frac{\partial \rho}{\partial t} \frac{\partial V}{\partial x} \frac{\partial^{2} V}{\partial x \partial t} d x+K \int_{0}^{1} \frac{\partial \rho}{\partial t} \theta \frac{\partial^{2} V}{\partial x \partial t} d x+K \int_{0}^{1} \rho \frac{\partial \theta}{\partial t} \frac{\partial^{2} V}{\partial x \partial t} d x-\int_{0}^{1} \frac{\partial^{2} v_{1}}{\partial t^{2}} \frac{\partial V}{\partial t} d x
\end{aligned}
$$


Applying (4.6) and (2.22) for $\rho,(2.24)$ for $\theta,(4.7)$ for $\partial V / \partial x$ and the Young inequality with a parameter $\varepsilon>0$ we obtain

$$
\begin{gathered}
\left|\int_{0}^{1} \frac{\partial \rho}{\partial t} \frac{\partial V}{\partial x} \frac{\partial^{2} V}{\partial x \partial t} d x\right| \leq \varepsilon \int_{0}^{1} \rho\left(\frac{\partial^{2} V}{\partial x \partial t}\right)^{2} d x+C\left\|\frac{\partial^{2} V}{\partial x^{2}}(t)\right\|^{2} \\
\left|K \int_{0}^{1} \frac{\partial \rho}{\partial t} \theta \frac{\partial^{2} V}{\partial x \partial t} d x\right| \leq C+\varepsilon \int_{0}^{1} \rho\left(\frac{\partial^{2} V}{\partial x \partial t}\right)^{2} d x \\
\left|K \int_{0}^{1} \rho \frac{\partial \theta}{\partial t} \frac{\partial^{2} V}{\partial x \partial t} d x\right| \leq \varepsilon \int_{0}^{1} \rho\left(\frac{\partial^{2} V}{\partial x \partial t}\right)^{2} d x+C\left\|\frac{\partial \theta}{\partial t}(t)\right\|^{2} \\
\left|\int_{0}^{1} \frac{\partial^{2} v_{1}}{\partial t^{2}} \frac{\partial V}{\partial t} d x\right| \leq C\left(\left\|\frac{\partial^{2} v_{1}}{\partial t^{2}}(t)\right\|^{2}+\left\|\frac{\partial V}{\partial t}(t)\right\|^{2}\right) .
\end{gathered}
$$

For sufficiently small $\varepsilon>0$ from (4.10) and (4.11)-(4.14) it follows that for $t \in] 0, T$ [ we have

$$
\begin{gathered}
\left\|\frac{\partial V}{\partial t}(t)\right\|^{2}+\int_{0}^{t}\left\|\frac{\partial^{2} V}{\partial x \partial t}(\tau)\right\|^{2} d \tau \leq C\left(1+\left\|\frac{\partial V}{\partial t}(0)\right\|^{2}+\int_{0}^{t}\left\|\frac{\partial \theta}{\partial t}(\tau)\right\|^{2} d \tau+\int_{0}^{t}\left\|\frac{\partial^{2} V}{\partial x^{2}}(\tau)\right\|^{2} d \tau\right. \\
\left.+\int_{0}^{t}\left\|\frac{\partial^{2} v_{1}}{\partial t^{2}}(\tau)\right\|^{2} d \tau+\int_{0}^{t}\left\|\frac{\partial V}{\partial t}(\tau)\right\|^{2} d \tau\right) .
\end{gathered}
$$

Taking into account (3.2), (3.6), and (3.7) from (2.31) we can easily conclude that

$$
\left\|\frac{\partial V}{\partial t}(0)\right\|=\left\|\rho_{0}^{\prime} V_{0}^{\prime}+\rho_{0} V_{0}^{\prime \prime}-K \rho_{0}^{\prime} \theta_{0}-K \rho_{0} \theta_{0}^{\prime}-\frac{\partial v_{1}}{\partial t}(0)\right\| \leq C,
$$

and using (2.20) and (4.1) we get that inclusion (4.6) is satisfied for the function $V$. In the similar way from (2.32) and (2.33) we obtain (4.6) for $W$ and $\theta$. for

Now, taking into account (4.6) and (2.20), we can introduce the following inequalities

$$
\eta \in\{V, W, \theta\}
$$

derived in [3] by the Nirenberg-Gagliardo inequality (2.44). 
Lemma 4.3 (see [3, Lemmas 2.2-2.4]). For $0<\alpha<1$ and $\varepsilon>0$, the function $\eta$ satisfies the inequalities

$$
\begin{aligned}
\left|\frac{\partial \eta}{\partial x}\right|_{0, Q_{T}} & \leq C|\eta|_{2+\alpha, 1+\alpha / 2, Q_{Q^{\prime}}}^{a} \\
\left|\frac{\partial \eta}{\partial x}\right|_{\alpha, \alpha / 2, Q_{T}} & \leq C\left(\varepsilon|\eta|_{2+\alpha, 1+\alpha / 2, Q_{T}}+\sup _{Q_{T}}\left|\frac{\partial \eta}{\partial t}\right|+1\right), \\
\left|\frac{\partial \eta}{\partial x}\right|_{\alpha, Q_{T}} & \leq C|\eta|_{2+\alpha, 1+\alpha / 2, Q_{T}}^{d}\left(|\eta|_{2+\alpha, 1+\alpha / 2, Q_{T}}+1\right)^{1-d},
\end{aligned}
$$

where $a=1 /(3+2 \alpha)$ and $d=\alpha /(2-\alpha)$. For $0<\alpha \leq 1 / 2$ it holds

$$
\left|\frac{\partial \eta}{\partial x}\right|_{\alpha, \alpha / 2, Q_{T}} \leq C|\eta|_{2+\alpha, 1+\alpha / 2, Q_{T}}^{b}
$$

where $b=(1+2 \alpha) /(3+2 \alpha)$.

\section{The proofs of Theorems 3.1 and 3.2}

The conclusions of Theorems 3.1 and 3.2 are immediate consequences of the following lemmas.

Lemma 5.1. It holds

$$
\rho, V, W, \theta \in C^{1 / 2,1 / 2}\left(\bar{Q}_{T}\right) .
$$

Moreover,

$$
v_{1}, \omega_{1} \in C^{1+1 / 2,1+1 / 2}\left(\bar{Q}_{T}\right)
$$

Proof. Taking into account (4.6) we get inclusion (5.1) for the functions $V, W$, and $\theta$ in the same way as for $\rho$ in (see [8, pages 54-55]). Using (3.1), (3.6), and (5.1) from (2.26), (2.27), (4.2), and (4.3) we get (5.2) immediately.

Lemma 5.2. For $0<\alpha<1$ and $\gamma=\min \{1 / 2, \alpha\}$ it holds

$$
\frac{\partial \rho}{\partial x} \in C^{\gamma, \gamma}\left(\dot{Q}_{T}\right)
$$

Proof. With the help of (5.1), (5.2), and (3.2) we obtain (5.3) in the similar way as in (see [8, pages 57-58]). 
Lemma 5.3. For $0<\alpha<1, \gamma=\min \{1 / 2, \alpha\}, a=1 /(3+2 \alpha)$, and $b=(1+2 \gamma) /(3+2 \gamma)$ the inequalities

$$
\begin{gathered}
|V|_{2+\gamma, 1+\gamma / 2, Q_{T}} \leq C\left(1+|\theta|_{1+\gamma, \gamma / 2, Q_{T}}\right), \\
|W|_{2+\gamma, 1+\gamma / 2, Q_{T}} \leq C, \\
|\theta|_{2+\gamma, 1+\gamma / 2, Q_{T}} \leq C\left(|V|_{2+\gamma, 1+\gamma / 2, Q_{T}}^{a}+|V|_{2+\gamma, 1+\gamma / 2, Q_{T}}^{b}+|V|_{2+\gamma, 1+\gamma / 2, Q_{T}}^{a+b}+|V|_{2+\gamma, 1+\gamma / 2, Q_{T}}^{2 a}+1\right)
\end{gathered}
$$

hold true.

Proof. We write (2.31), (2.32), and (2.33) in the form

$$
\begin{gathered}
\frac{\partial V}{\partial t}-\rho \frac{\partial^{2} V}{\partial x^{2}}-\frac{\partial \rho}{\partial x} \frac{\partial V}{\partial x}=-K \frac{\partial \rho}{\partial x} \theta-K \rho \frac{\partial \theta}{\partial x}-\frac{\partial v_{1}}{\partial t} \\
\frac{\partial W}{\partial t}-A \rho \frac{\partial^{2} W}{\partial x^{2}}-A \frac{\partial \rho}{\partial x} \frac{\partial W}{\partial x}+A \frac{W}{\rho}=-A \frac{\omega_{1}}{\rho}-\frac{\partial \omega_{1}}{\partial t} \\
\frac{\partial \theta}{\partial t}-D \rho \frac{\partial^{2} \theta}{\partial x^{2}}-D \frac{\partial \rho}{\partial x} \frac{\partial \theta}{\partial x}=-K \rho \theta \frac{\partial V}{\partial x}-K \theta \frac{\mu}{l}+\rho\left(\frac{\partial V}{\partial x}\right)^{2}+2 \frac{\partial V}{\partial x} \frac{\mu}{l}+\frac{1}{\rho}\left(\frac{\mu}{l}\right)^{2} \\
+\rho\left(\frac{\partial W}{\partial x}\right)^{2}+2 \frac{\partial W}{\partial x} \frac{v}{l}+\frac{1}{\rho}\left(\frac{v}{l}\right)^{2}+\frac{1}{\rho}\left(W+\omega_{1}\right)^{2},
\end{gathered}
$$

and we consider them as parabolic equations for $V, W$, and $\theta$, respectively, with Hölder continuous coefficients with exponent $\gamma=\min \{1 / 2, \alpha\}$. Taking into account the compatibility conditions (3.8)-(3.10), (2.14), and $|f g|_{\alpha, \alpha / 2} \leq|f|_{0}|g|_{\alpha, \alpha / 2}+|f|_{\alpha, \alpha / 2}|g|_{0}$, from a parabolic theory (see [7, Theorems 5.2 and 5.3]) we conclude that the solutions $V, W$, and $\theta$ satisfy the following inequalities

$$
\begin{aligned}
|V|_{2+\gamma, 1+\gamma / 2, Q_{T}} \leq C & \left(\left|\frac{\partial \rho}{\partial x}\right|_{\gamma, \gamma / 2, Q_{T}}|\theta|_{0, Q_{T}}+\left|\frac{\partial \rho}{\partial x}\right|_{0, Q_{T}}|\theta|_{\gamma, \gamma / 2, Q_{T}}+|\rho|_{\gamma, \gamma / 2, Q_{T}}\left|\frac{\partial \theta}{\partial x}\right|_{0, Q_{T}}\right. \\
& \left.+|\rho|_{0, Q_{T}}\left|\frac{\partial \theta}{\partial x}\right|_{\gamma, \gamma / 2, Q_{T}}+\left|\frac{\partial v_{1}}{\partial t}\right|_{\gamma, \gamma / 2, Q_{T}}+\left|V_{0}\right|_{2+\gamma, \Omega}\right), \\
|W|_{2+\gamma, 1+\gamma / 2, Q_{T}} \leq & \left(\left|\frac{1}{\rho}\right|_{0, Q_{T}}\left|\omega_{1}\right|_{\gamma, \gamma / 2, Q_{T}}+\left|\frac{1}{\rho}\right|_{\gamma, \gamma / 2, Q_{T}}\left|\omega_{1}\right|_{0, Q_{T}}+\left|\frac{\partial \omega_{1}}{\partial t}\right|_{\gamma, \gamma / 2, Q_{T}}+\left|W_{0}\right|_{2+\gamma, \Omega}\right),
\end{aligned}
$$




$$
\begin{aligned}
|\theta|_{2+\gamma, 1+\gamma / 2, Q_{T} \leq C} \leq & |\rho \theta|_{0, Q_{T}}\left|\frac{\partial V}{\partial x}\right|_{\gamma, \gamma / 2, Q_{T}}+|\rho \theta|_{\gamma, \gamma / 2, Q_{T}}\left|\frac{\partial V}{\partial x}\right|_{O_{,} Q_{T}}+|\theta|_{\gamma, \gamma / 2, Q_{T}} \\
& +\left|\rho \frac{\partial V}{\partial x}\right|_{0, Q_{T}}\left|\frac{\partial V}{\partial x}\right|_{\gamma, \gamma / 2, Q_{T}}+\left|\rho \frac{\partial V}{\partial x}\right|_{\gamma, \gamma / 2, Q_{T}}\left|\frac{\partial V}{\partial x}\right|_{0, Q_{T}}+\left|\frac{\partial V}{\partial x}\right|_{\gamma, \gamma / 2, Q_{T}} \\
& +\left|\frac{1}{\rho}\right|_{\gamma, \gamma / 2, Q_{T}}+\left|\rho \frac{\partial W}{\partial x}\right|_{0, Q_{T}}\left|\frac{\partial W}{\partial x}\right|_{\gamma, \gamma / 2, Q_{T}}+\left|\frac{\partial W}{\partial x}\right|_{\gamma, \gamma / 2, Q_{T}} \\
& +\left|\rho \frac{\partial W}{\partial x}\right|_{\gamma, \gamma / 2, Q_{T}}\left|\frac{\partial W}{\partial x}\right|_{0, Q_{T}}+\left|\frac{W}{\rho}\right|_{0, Q_{T}}|W|_{\gamma, \gamma / 2, Q_{T}} \\
& \left.+\left|\frac{W}{\rho}\right|_{\gamma, \gamma / 2, Q_{T}}|W|_{0, Q_{T}}+\left|\frac{\omega_{1}^{2}}{\rho}\right|_{\gamma, \gamma / 2, Q_{T}}+\left|\theta_{0}\right|_{2+\gamma, \Omega}\right) .
\end{aligned}
$$

Using the inequalities

$$
|f|_{0_{,} Q_{T}} \leq|f|_{\gamma, \gamma / 2, Q_{T}} \leq|f|_{\gamma, \gamma, Q_{T}}
$$

and (3.6), (3.2), and (5.1)-(5.3), from (5.8)-(5.9) we get easily (5.4) and (5.5). With the help of (4.18), (4.21) for $\eta=W$ and (5.5) from (5.10) it follows

$$
|\theta|_{2+\gamma, 1+\gamma / 2, Q_{T}} \leq C\left(\left|\frac{\partial V}{\partial x}\right|_{\gamma, \gamma / 2, Q_{T}}+\left|\frac{\partial V}{\partial x}\right|_{0, Q_{T}}+\left|\frac{\partial V}{\partial x}\right|_{\gamma, \gamma / 2, Q_{T}}\left|\frac{\partial V}{\partial x}\right|_{0, Q_{T}}+\left|\frac{\partial V}{\partial x}\right|_{0, Q_{T}}^{2}+1\right) .
$$

Using (4.18) and (4.21) for $\eta=V$ we get (5.6) immediately.

Lemma 5.4. For $r$ from Lemma 5.2 the estimations

$$
\begin{gathered}
|V|_{2+\gamma, 1+\gamma / 2, Q_{T}} \leq C, \\
|\theta|_{2+\gamma, 1+\gamma / 2, Q_{T}} \leq C, \\
|\rho|_{1+\gamma, Q_{T}} \leq C, \\
\left|v_{1}\right|_{2+\gamma, 1+\gamma / 2, Q_{T}} \leq C, \quad\left|\omega_{1}\right|_{2+\gamma, 1+\gamma / 2, Q_{T}} \leq C
\end{gathered}
$$

hold true.

Proof. For $\eta=\theta$ and $0<\alpha<1$ from (4.19) we can conclude that

$$
|\theta|_{1+\alpha, \alpha / 2, Q_{T}} \leq C\left(|\theta|_{2+\alpha, 1+\alpha / 2, Q_{T}}+1\right),
$$

and from (5.4) it follows

$$
|V|_{2+\gamma, 1+\gamma / 2, Q_{T}} \leq C\left(|\theta|_{2+\gamma, 1+\gamma / 2, Q_{T}}+1\right) .
$$


Inserting (5.6) on the right-hand side of (5.18) we obtain

$$
|V|_{2+\gamma, 1+\gamma / 2, Q_{T}} \leq C\left(|V|_{2+\gamma, 1+\gamma / 2, Q_{T}}^{a}+|V|_{2+\gamma, 1+\gamma / 2, Q_{T}}^{b}+|V|_{2+\gamma, 1+\gamma / 2, Q_{T}}^{a+b}+|V|_{2+\gamma, 1+\gamma / 2, Q_{T}}^{2 a}+1\right),
$$

where $a, b, a+b, 2 a \in] 0,1[$. Applying the Young inequality with a parameter $\varepsilon>0$ we obtain

$$
|V|_{2+\gamma, 1+\gamma / 2, Q_{T}} \leq C\left(\varepsilon|V|_{2+\gamma, 1+\gamma / 2, Q_{T}}+1\right),
$$

and hence (5.13). Using this result from (5.6) follows (5.14). From (2.30) we get

$$
\left|\frac{\partial \rho}{\partial t}\right|_{\gamma, Q_{T}} \leq\left|\rho^{2}\right|_{0, Q_{T}}\left|\frac{\partial V}{\partial x}\right|_{\gamma, Q_{T}}+\left|\rho^{2}\right|_{\gamma, Q_{T}}\left|\frac{\partial V}{\partial x}\right|_{0, Q_{T}}+\left|\frac{\mu}{l} \rho\right|_{\gamma, Q_{T}} .
$$

Using (4.20) and (5.13) for $V$, the inequality $|\rho|_{\gamma, Q_{T}} \leq|\rho|_{\gamma, \gamma, Q_{T}},(5.1)$ and (3.1) we obtain

$$
\left|\frac{\partial \rho}{\partial t}\right|_{\gamma, Q_{T}} \leq C
$$

and with the help of (5.3) we get (5.15). Notice that from (5.15) follows

$$
\int_{0}^{x} \rho^{-1} d \xi \in C^{\gamma, \gamma / 2}\left(\bar{Q}_{T}\right)
$$

and using (5.13) and (3.1) from (4.2) and (2.26) we obtain

$$
\begin{aligned}
&\left|\frac{\partial v_{1}}{\partial t}\right|_{\gamma, \gamma / 2, Q_{T}} \leq\left|\left(\frac{\mu}{l}\right)^{\prime}+\left(\frac{\mu}{l}\right)^{2} \int_{0}^{x} \frac{1}{\rho} d \xi\right|_{\gamma, \gamma / 2, Q_{T}}+\left|\frac{\mu}{l} V\right|_{\gamma, \gamma / 2, Q_{T}}+\left|\mu_{0}^{\prime}\right|_{\gamma / 2,] 0, T[} \leq C, \\
&\left|\frac{\partial^{2} v_{1}}{\partial x^{2}}\right|_{\gamma, \gamma / 2, Q_{T}} \leq\left|\frac{\mu}{l \rho}\right|_{\gamma, \gamma / 2, Q_{T}}\left|\frac{\partial \rho}{\partial x}\right|_{0, Q_{T}}+\left|\frac{\mu}{l \rho}\right|_{0, Q_{T}}\left|\frac{\partial \rho}{\partial x}\right|_{\gamma, \gamma / 2, Q_{T}}+\left|\frac{\mu}{l}\right|_{\gamma,] 0, T[} \leq C .
\end{aligned}
$$

Taking into account (5.2) it is evident that the inequality

$$
\left|v_{1}\right|_{2+\gamma, 1+\gamma / 2, Q_{T}} \leq C
$$

is satisfied. From (2.27) and (4.3) follows the same estimation for the function $\omega_{1}$.

Now, from the above estimations we derive the conclusion that if $\alpha \leq 1 / 2$ then $\alpha=\gamma$ and Lemmas 5.1-5.4 are the proofs of Theorems 3.2 and 3.1. If $\alpha>1 / 2$ we have

$$
V, W, \theta, v_{1}, \omega_{1} \in C^{2+1 / 2,1+1 / 4}\left(\bar{Q}_{T}\right), \quad \rho \in C^{1+1 / 2}\left(\bar{Q}_{T}\right)
$$


Lemma 5.5. For $1 / 2<\alpha<1$ we have

$$
\begin{gathered}
\rho, V, W, \theta \in C^{\alpha, \alpha}\left(\bar{Q}_{T}\right), \\
v_{1}, \omega_{1} \in C^{1+\alpha, 1+\alpha}\left(\bar{Q}_{T}\right), \\
\frac{\partial \rho}{\partial x} \in C^{\alpha, \alpha}\left(\dot{Q}_{T}\right) .
\end{gathered}
$$

Proof. Inclusions (5.27) follows directly from (5.26). Using this result from (2.26), (2.27), (4.2), and (4.3) we get (5.28). Estimation (5.29) is proved in (see [8, pages 57-58]).

Lemma 5.6. For $1 / 2<\alpha<1$ the estimations

$$
\begin{gathered}
|V|_{2+\alpha, 1+\alpha / 2, Q_{T}} \leq C, \\
|W|_{2+\alpha, 1+\alpha / 2, Q_{T}} \leq C, \\
|\theta|_{2+\alpha, 1+\alpha / 2, Q_{T}} \leq C, \\
|\rho|_{1+\alpha, Q_{T}} \leq C . \\
\left|v_{1}\right|_{2+\alpha, 1+\alpha / 2, Q_{T}} \leq C, \quad\left|\omega_{1}\right|_{2+\alpha, 1+\alpha / 2, Q_{T}} \leq C
\end{gathered}
$$

are true.

Proof. We consider (2.31)-(2.33) again as parabolic equations for $V, W$, and $\theta$, respectively, with Hölder continuous coefficients with exponent $\alpha$. In the same way as before from (5.8) and (5.9) we get

$$
\begin{gathered}
|V|_{2+\alpha, 1+\alpha / 2, Q_{T}} \leq C\left(1+|\theta|_{2+\alpha, 1+\alpha / 2, Q_{T}}\right), \\
|W|_{2+\alpha, 1+\alpha / 2, Q_{T}} \leq C
\end{gathered}
$$

and with the help of (5.26) from (5.10) we obtain

$$
|\theta|_{2+\alpha, 1+\alpha / 2, Q_{T}} \leq C\left(1+\left|\frac{\partial V}{\partial x}\right|_{\alpha, \alpha / 2, Q_{T}}\right) .
$$

Inserting (5.37) in (5.35), using (4.19) and (5.26) for the function $V$, we obtain (5.30). With the help of (4.19) and (5.30) from (5.37) it follows (5.32). In the same way as before we get

$$
\left|\frac{\partial \rho}{\partial t}\right|_{\alpha, Q_{T}} \leq\left|\rho^{2}\right|_{\alpha, Q_{T}}\left|\frac{\partial V}{\partial x}\right|_{0, Q_{T}}+\left|\rho^{2}\right|_{0, Q_{T}}\left|\frac{\partial V}{\partial x}\right|_{\alpha, Q_{T}}+\left|\frac{\mu}{l} \rho\right|_{\alpha, Q_{T}} .
$$

Because of (5.27), (5.30), and (4.20) for $\eta=V$ we obtain

$$
\left|\frac{\partial \rho}{\partial t}\right|_{\alpha, Q_{T}} \leq C
$$


and using (5.29) we have (5.33). Taking into account (5.33) in the same way as in Lemma 5.4 we get (5.34).

\section{References}

[1] N. Mujaković, "One-dimensional flow of a compressible viscous micropolar fluid: a local existence theorem," Glasnik Matematički, vol. 33, no. 1, pp. 71-91, 1998.

[2] N. Mujaković, "One-dimensional flow of a compressible viscous micropolar fluid: a global existence theorem," Glasnik Matematički, vol. 33, no. 2, pp. 199-208, 1998.

[3] N. Mujaković, "One-dimensional flow of a compressible viscous micropolar fluid: regularity of the solution," Radovi Matematički, vol. 10, no. 2, pp. 181-193, 2001.

[4] N. Mujaković, "Non-homogeneous boundary value problem for one-dimensional compressible viscous micropolar fluid model: a local existence theorem," Annali dell'Universitá di Ferrara, vol. 53, no. 2, pp. 361-379, 2007.

[5] N. Mujaković, "Non-homogeneous boundary value problem for one-dimensional compressible viscous micropolar fluid: a global existence theorem," to appear in Mathematical Inequalities $\mathcal{E}$ Applications.

[6] B. L. Roždestvenskiř and N. N. Janenko, Systems of Quasilinear Equations and Their Applications to Gas Dynamics, vol. 55 of Translations of Mathematical Monographs, American Mathematical Society, Providence, RI, USA, 1983.

[7] O. A. Ladyzhenskaya, V. A. Solonnikov, and N. N. Uraltseva, Linear and Quasi-Linear Equations of Parabolic Type, American Mathematical Society, Providence, RI, USA, 1968.

[8] S. N. Antontsev, A. V. Kazhikhov, and V. N. Monakhov, Boundary Value Problems in Mechanics of Nonhomogeneous Fluids, vol. 22 of Studies in Mathematics and Its Applications, North-Holland, Amsterdam, The Netherlands, 1990.

[9] J. L. Lions and E. Magenes, Non-Homogeneous Boundary Value Problems and Applications, vol. 1 of Die Grundlehren der Mathematischen Wissenschaften, Springer, Berlin, Germany, 1972. 